\title{
Complex Permittivity to Detect Lead in Soil with Various Salt Forms and Combinations
}

\author{
Samer Al Martini ${ }^{1, *}$, and Julie Q. Shang ${ }^{2}$ \\ ${ }^{1}$ Department of Civil Engineering, College of Engineering, Abu Dhabi University, Abu Dhabi, UAE \\ ${ }^{2}$ Department of Civil Engineering, Western University, London, Ontario, Canada
}

\begin{abstract}
The response of soil-water systems to an external electric field can be described as polarization and conduction. Polarization represents the electric charges stored in a material, whereas conduction is the ability of a material to conduct free charges. The combined effects of polarization and conduction can be expressed in terms of complex permittivity. Since the complex permittivity of a soil-water system is a function of soil properties, this research was undertaken to detect soil contamination using the complex permittivity measurements. The system used in this investigation to measure the complex permittivity of a lead contaminated soil consisted of an Automatic Network Analyzer (ANA), a set of coaxial cables, a personal computer (PC) for data processing and a coaxial sample holder. Two sample holders were first used: long holder and short holder. It was found that the short holder can give reliable and close measurement to the long holder. The short holder was later adopted. Soil samples were prepared in the lab and mixed with lead nitrate solutions and with hybrid blend of lead nitrate and lead chloride solutions. The relationship between the complex permittivity and lead concentration was investigated. A chemical analysis of the pore fluids for all lead contaminated soil samples was conducted to trace the presence of lead in soil. It was found that the complex permittivity is affected by lead concentration in soil, as the real part decreased and the imaginary part increased with an increase in lead concentration. Lead was not detected in the soil pore water for the soil tested, which suggested that lead was immobilized by precipitation and the adsorption mechanism.
\end{abstract}

\section{Introduction}

The last century witnessed vast industrial revolution that resulted in global environmental contamination problems. Many industries all over the world, and especially in developed countries, generate wastewater daily, which contains high levels of metals. In the past, at the start of the industrial revolution, any damage that heavy metals could have caused to the environment was not known. Many industries discharged their wastewaters directly to rivers and lakes causing pollution of water and soil. Eventually, the contamination is transferred to humans, wildlife and vegetation. The environmental problem has become an

* Corresponding author: samer.almartini@adu.ac.ae 
important issue in the world. Strict environmental regulations have been established in order to protect the environment from pollution. Researchers recently focused on research contamination issues and monitoring systems [1-4].

Heavy metals in general and lead in particular are the most dangerous contaminants in soil. Heavy metals usually have very low mobility due to their strong affinity for the solid phase [5]. Due to its low mobility, when introduced to soil, lead usually accumulates in the upper layer of the ground surface where vegetables and grass grow. This highlights the danger of lead contamination in several ways including the pathway from vegetation to humans, animals and direct contact [6].

It is essential that lead contamination be monitored. Monitoring includes gathering information about the extent of pollution in the area, so that the environment can be protected controlled, and lead levels maintained within the allowed limits using government regulations and standards. Many countries have put standards and regulations of heavy metals and lead in place. What remains to be seen is how these regulations can be followed and controlled.

The first step is to provide a comprehensive monitoring system which most countries do not have. This is due to the lack of a means of lead monitoring and because this issue has not been given high priority. Consequently, the level of heavy metals contamination, and lead specifically, is not known precisely [7]. It has now become urgent that monitoring systems be developed and installed beginning with regulations and ending with the development of a means to measure lead levels in the ambient environment. The monitoring system includes off situ and in situ systems. Many companies have taken important steps to develop lead monitoring systems. Extensive research work has been done and reported in the literature to achieve this objective.

A soil is a three-phase system (i.e. solids, liquid and gas). Each component of soil has a different dielectric behaviour and thus different dielectric constant values. As a result, the complex permittivity of the soil water mixture is a function of the dielectric properties of all its constituents. Water is present in the soil as free water and adsorbed water. The microwave aquametry studies the interaction between the soil-water system and the incident electromagnetic waves [8]. The complex permittivity of soil was investigated by several researchers to monitor soil contamination $[1,2,9-11]$. The complex permittivity $\left(\varepsilon^{*}\right)$ consists of two terms real part and imaginary part.

In the course of the current research, the soil complex permittivity method is investigated and evaluated for detecting lead contamination in soil. The system used to measure the soil complex permittivity consists of coaxial cable, sample holder, analyzer, and computer. The soil is placed in a coaxial holder to measure its complex permittivity. Because several researchers have had success, when using the coaxial holder to characterize soil properties $[12,13]$, this holder was used in this investigation. Two lead salts $(\mathrm{PbCl} 2$, $\mathrm{Pb}(\mathrm{NO} 3) 2$ ) at different concentrations were mixed with soil. The lead concentrations were selected based on Ontario Ministry of Environment and Energy [11] for soil (200 ppm $1000 \mathrm{ppm})$. The lowest concentration was chosen according to the MOEE Guideline [14] for residential areas, while the highest concentration was based on the allowable lead concentration for industrial areas. The objective of this paper was to investigate the relationship between soil complex permittivity and lead concentrations in various forms, which could be used as an indicator to detect soil contamination with lead.

\section{Experimental program}

The Halton Till soil was used in this investigation. This soil is a brown to gray clayey silt with illite, chlorite, and smectite being the major clay minerals. The non-clay constituents include quartz, carbonates, and feldspar [8]. Lead nitrate salt ( $\mathrm{Pb}(\mathrm{NO} 3) 2)$ was dissolved in 
water at five different concentrations. In addition, multiple salt solutions were prepared using two lead salts $((\mathrm{Pb}(\mathrm{NO} 3) 2)$ and $(\mathrm{PbCl} 2))$ to study the possibility of tracing lead contamination in various forms. Lead solutions were mixed with soil sample.

The soil was placed in the oven at a temperature of 105 oC for 24 hours. The soil was then pulverized and sieved through sieve \#4. The pulverized soil was mixed with distilled water or lead solutions with a moisture content of $19 \pm 1 \%$ (the mass ratio of water to dry soil). The wet soil was placed in a plastic bag and sealed to prevent evaporation, and it was left overnight. A small specimen was taken from the bag for the moisture content measurement. The empty sample cell was weighed and its weight was recorded. The soil sample was compacted in the cell in three layers with three blows for each layer using a special apparatus designed by Scholte [13]. A Proctor standard hammer was employed to compact the tested soil with a weight of $24.5 \mathrm{~N}$ and at a fall height of 0.305 providing 76.4 $\mathrm{KJ} / \mathrm{m} 3$ compacting energy per fall [13]. The compacted sample cell was weighed and the mass was recorded. The bulk density of the sample was calculated.

The system used to measure the complex permittivity of a lead contaminated soil consists of an Automatic Network Analyzer (ANA), a set of coaxial cables, a personal computer (PC) for data processing and a coaxial sample holder (see Fig. 1). The soil sample was installed in the holder for complex permittivity measurements. The measurements were performed quickly to minimize any drying effect so that the measured complex permittivity reflects the real soil conditions. It took experienced personnel less than one minute to perform one measurement.

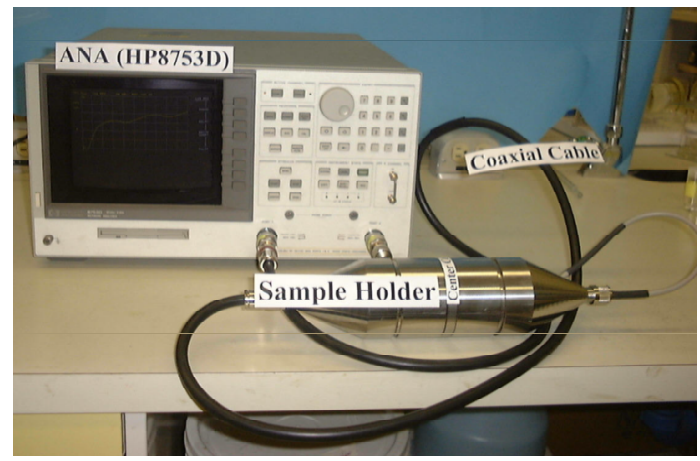

Fig. 1. Complex permittivity measurement system

\section{Results and discussion}

\subsection{Complex permittivity of soil sample mixed with distilled water}

Fig. 2 shows six repetitive measurements of the complex permittivity for a soil mixed with distilled water. Fig. 2(a) shows results for real part and Fig. 2 (b) shows results for the imaginary part. These Fig.s show that the six results are highly consistent, which indicates that the system can produce reliable repetitive measurements. The longitudinal resonance is observed at the frequency where a discontinuity in the real and imaginary parts of the complex permittivity occurs. The resonance frequency occurs at a range between 910 and $930 \mathrm{MHz}$. 


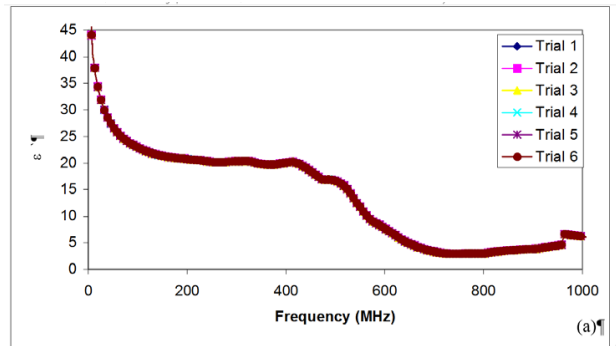

(a) Real part

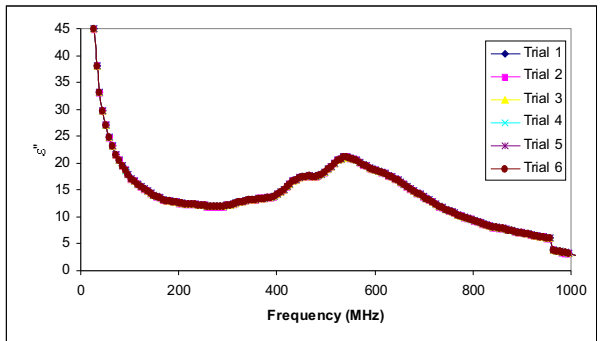

(b) Imaginary part

Fig. 2. Repeatability of complex permittivity measurements for soil mixed with distilled water using short holder

\subsection{Complex permittivity of soil samples mixed with lead nitrate solutions}

Five soil samples of Halton Till were prepared with five different concentrations of lead nitrate solution $(\mathrm{c}=0.8,1.6,4.5,8.1,14.3 \mathrm{~g} / \mathrm{l})$. The complex permittivity of soil samples were measured in both short holder $(1=261 \mathrm{~mm})$ and long holder $(1=321 \mathrm{~mm})$. The results for both real and imaginary parts of the complex permittivity were plotted over frequencies 0.3 to $1000 \mathrm{MHz}$ for each compacted soil sample. Fig. 3 (a) shows the real part (long-short holders) of complex permittivity for soil samples mixed with lead nitrate solutions $(\mathrm{c}=8.1$, g/l), and Fig. 3(b) shows the imaginary part for the same soil sample. As shown in Fig. 3(a), the relative permittivity in low frequency was high, which is attributed to the polarization of the electrical double layer [15]. When the polarization of the adsorbed electrical double layer can no longer change quickly enough to reach equilibrium with an applied external alternating electric field, dielectric dispersion occurs [13]. The dielectric dispersion begins where the permittivity starts to drop. As indicated by Scholte [13], the dielectric dispersion usually occurs in frequencies of 200 to $500 \mathrm{MHz}$ for Halton Till. The dielectric dispersion was noticed at frequencies of 395 and $418 \mathrm{MHz}$ for long and short holders, respectively. The relative permittivity was approximately constant over the frequency range 200 to 400 $\mathrm{MHz}$ for both long and short holders. This permittivity is known as the static dielectric constant $\left(\varepsilon_{\mathrm{s}}\right)$. After these frequencies (the dielectric dispersion) the relative permittivity decreased until it reached the lowest values $\left(\varepsilon^{\prime}{ }_{\infty}\right)$. These Fig.s show that the dielectric dispersion for the short holder occurred at higher frequencies than for the long holder. The loss factor was lower in the short holder than in the long holder (Fig. 3(b)).

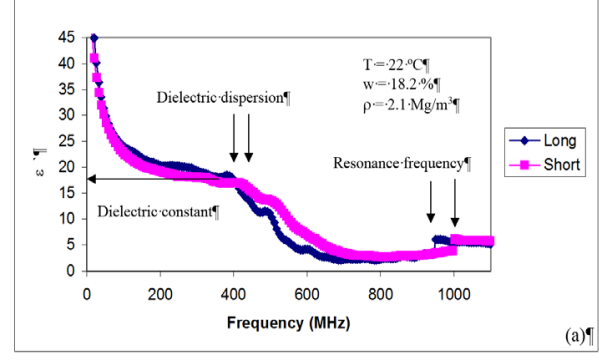

(a) Real part

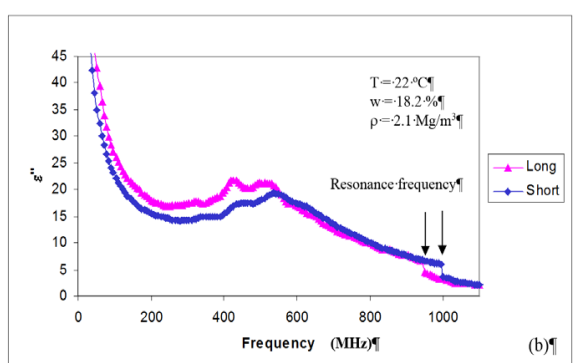

(b) Imaginary part

Fig. 3. Permittivity measurements of soil sample mixed with lead nitrate solution ( $c=8.1 \mathrm{~g} / \mathrm{l})$ for long and short holders 
Fig. 4(a) shows the relationship between the dielectric constant and lead nitrate concentrations for the short holder. This Fig. illustrates that the dielectric constant decreased linearly with an increase of lead nitrate concentration. Fig. 4(b) shows the relationship between the loss factor and the lead nitrate concentrations when the short holder was used. This Fig. shows that the loss factor increased with an increase of lead nitrate concentration. This indicates that using the short holder for measuring the complex permittivity can distinguish the change in lead nitrate concentrations in soil.

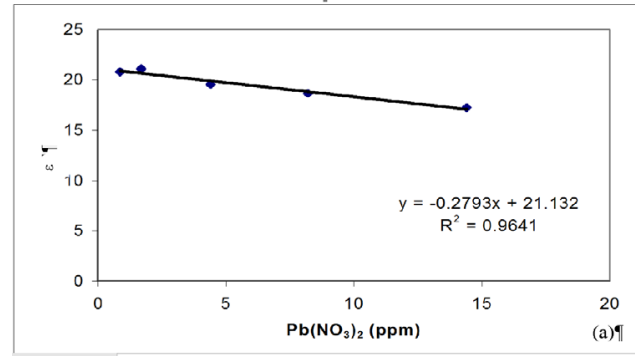

(a) Real part at $\mathrm{f}=200-400 \mathrm{MHz}$

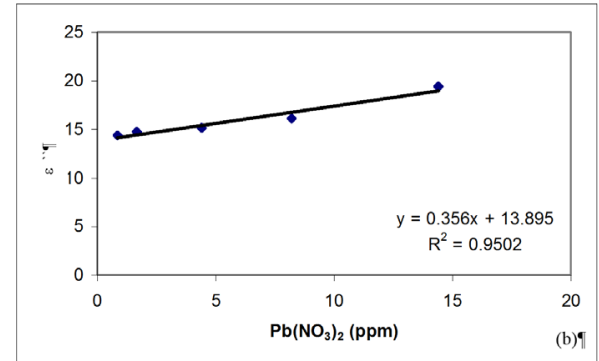

(b) Imaginary part at $\mathrm{f}=150-250 \mathrm{MHz}$

Fig. 4. The relationship between the complex permittivity and lead nitrate concentrations (short holder)

\subsection{Complex permittivity of soil mixed with $\mathrm{PbCl} 2+\mathrm{Pb}(\mathrm{NO}) 2$ solutions}

Fig. 5 shows results for soil sample mixed with $\mathrm{PbCl}_{2}+\mathrm{Pb}\left(\mathrm{NO}_{3}\right)_{2}$ solutions. Fig. 5a presents the relationship between the lead concentrations and the real relative permittivity at the dispersion frequency $(320 \mathrm{MHz})$. As shown in this Fig. 5, the real relative permittivities decreased when salt concentrations increased. The relationship was linear with $\mathrm{R}^{2}=0.729$. Fig. $5 \mathrm{~b}$ presents the relationship between the imaginary part and lead concentrations at a frequency of $200 \mathrm{MHz}$. They are also linearly related. The imaginary part increased with an increase in lead salt with $\mathrm{R}^{2}=0.82$. Fig. $5 \mathrm{c}$ shows the relationship between the imaginary and relative real permittivity. They are linearly related with $\mathrm{R}^{2}=0.82$.

\section{Summary and conclusions}

Lead contaminated soil samples were prepared using aqueous solutions for two salts (lead nitrate and lead chloride). These two salts were chosen based on their solubility in water. Lead nitrate has very high water solubility and lead chloride has moderate water solubility. In order to detect lead contamination in soil in various forms, aqueous solution with blends of lead nitrate and lead chloride were prepared in five different concentration and mixed with soil. The complex permittivity of the lead contaminated soil samples was measured in order to study the capability of the complex permittivity method for detecting lead in soil water systems. The analysis of the pore fluid chemistry for the lead contaminated soil samples tested herein showed the absence of lead in the squeezed pore water. This suggests that lead is immobilized and retained on the soil particles by the mechanisms of adsorption, precipitation and exchange.

It was observed that complex permittivities are sensitive to changes in lead salt concentrations in soil. In general, the relative permittivity at dielectric dispersion frequency decreased, whereas, the loss factor at $200 \mathrm{MHz}$ increased with an increase in lead concentration. This indicates that complex permittivity can be used to monitor lead 
concentration in soil. The results also indicate that this method can be used as a fast and nondestructive method to detect lead in contaminated sites.

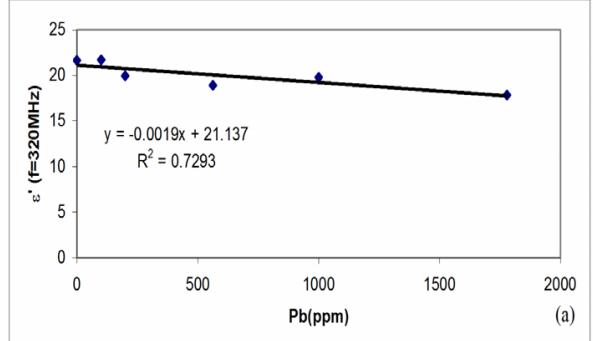

a) $\varepsilon^{\prime}$ at dielectric dispersion $(\mathrm{f}=320 \mathrm{MHz})$ versus concentration

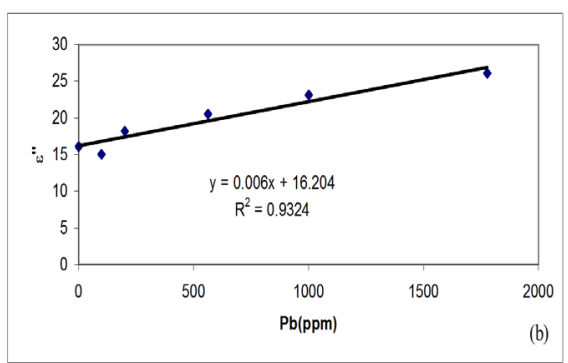

b) $\varepsilon "$ at $(\mathrm{f}=200 \mathrm{MHz})$ versus concentration

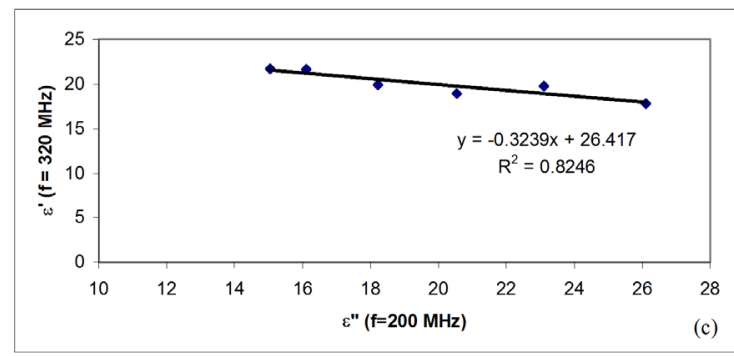

c) $\varepsilon$ ' at dielectric dispersion $(\mathrm{f}=320 \mathrm{MHz})$ versus $\varepsilon$ " at $(\mathrm{f}=200 \mathrm{MHz})$

Fig. 5. Halton Till mixed with lead chloride and lead nitrate $\left(\mathrm{PbCl}_{2}+\mathrm{Pb}\left(\mathrm{NO}_{3}\right)_{2}\right)$ complex permittivity relationships

\section{References}

[1] Q.J. Shang, K.R. Rowe, W. J. Scholte, Soil characterization using static conductivity and permittivity, Canadian Geotechnical Conference, Calgary, Alberta, 968-975 (2001)

[2] Q.J. Shang, W.J. Scholte, K.R. Rowe , Multiple linear regression of complex permittivity of halton till at frequency range from $200 \mathrm{MHz}$ to $400 \mathrm{MHz}$, Subsurface Sensing Technologies and Applications, 1(3), 337-356 (2000)

[3] S. Al-Martini, Q.J. Shang, Complex permittivity measurement system for detection of soil contamination, Alhosn University Journal of Engineering and Applied Science 4(1), 73-88 (2011)

[4] S. Al-Martini, Q.J. Shang, Complex permittivity of lead in contaminated soils, Proc. of GeoMontreal 2013, Montreal, Canada, (2013)

[5] A. Kabata-Pendias, Trace Elements in Soils and Plants, $3^{\text {rd }}$ ed., CRC Press, (2001)

[6] R. Wainner, T. Harmon, R. Miziolek, L. Mc Nesby, D. French, Analysis of environmental lead contamination, Spectrochimica Acta Part, B56, 777-793 (2001)

[7] A. Karivelil, Kinetics of Lead(II) Biosorption by Calcium Alginate Biopolymers, M.E.Sc. Thesis, The University of Western Ontario, (1995)

[8] A. Kraszewski, Microwave Aquametry, IEEE Press, New York, (1997)

[9] Q.J. Shang, K.Y. Lo, I. I. Inculet, Polarization and conduction of clay- water electrical systems, Journal of Geotechnical Engineering, 121(3), 243-248 (1995) 
[10] N.R. Peplinski, F.T. Ulaby, M.C. Dobson, Dielectric properties of soils in the $0.3-1.3$ GHz. range, IEEE Transactions on Geoscience and Remote Science, 33(3), 803-807 (1995)

[11] R.K. Rowe, J. Q. Shang, Y. Xie, Complex permittivity measurement system for detecting soil contamination, Canadian Geotechnical Journal, 38, 498-506 (2001)

[12] J.A. Umana, Measurement of dielectric permittivity of pavement materials, M.E.Sc Thesis, University of Western Ontario, (1998)

[13] J.W. Scholte, The complex permittivity of compacted halton till, M.E.Sc Thesis, University of Western Ontario, (1999)

[14] MOE, Guideline for use at contaminated sites in Ontario, Queen's Printer for Ontario, PIBS 3161E01, (1996)

[15] J.Q. Shang, J.A. Umana, F.M. Bartlett, J.R. Rossiter, Measurement of complex permittivity of asphalt pavement materials, Journal of Transportation Engineering, 125(4), 347-356 (1999) 